\title{
Steady shearing flows of deformable, inelastic spheres
}

\author{
Diego Berzi, $*^{a}$ and James T. Jenkins ${ }^{b}$ \\ Received (in $X X X, X X X)$ Xth $X X X X X X X X X 20 X X$, Accepted Xth $X X X X X X X X X 20 X X$ \\ DOI: 10.1039/b000000x
}

\begin{abstract}
5 We extend models for granular flows based on the kinetic theory beyond the critical volume fraction at which a rate-independent contribution to the stresses develops. This involves the incorporation of a measure of the duration of the particle interaction before and after this volume fraction. At volume fractions less than the critical, the stress components contain contributions from momentum exchanged in collisions that are influenced by the particle elasticity. At volume fractions greater than the critical, the 10 stress components contain both static contributions from particle elasticity and dynamic contributions from the momentum transfer associated with the release of elastic energy by the breaking of force chains. A simple expression for the duration of a collision before and after the critical volume fraction permits a smooth transition between the two regimes and predictions for the components of the stress in steady, homogeneous shearing that are in good agreement with the results of numerical simulations. Application

15 of the theory to steady, inhomogeneous flows reproduce the features of such flows seen in numerical simulations and physical experiments.
\end{abstract}

\section{Introduction}

Granular materials that are comprised of nearly spherical particles exhibit behaviour that ranges from that of a gas to that of a solid, 20 depending on the nature of the interaction between the grains. These interactions depend upon the volume fraction of the solid, and the volume fraction is sensitive to the loading applied to the aggregate. The range of behaviours that granular materials exhibit parallels that seen in other systems of soft matter, such as colloids 25 and non-Brownian suspensions. ${ }^{1,2}$

In shearing flows driven, for example, by the relative motion of two rigid, bumpy boundaries at volume fractions less than about 0.49 , the spheres interact through collisions that can be regarded as instantaneous, binary, and uncorrelated. In this case, 30 methods from the kinetic theory of dense gases that take into account the energy lost in a collision,,$^{3-5}$ can be used to predict the relationship between the forces necessary to maintain the flow and the rate of shearing. When the influence of the boundaries is negligible, both the pressure and the shear stress are quadratic in 35 the shear rate; as a consequence, the strength of the velocity fluctuations, the analogue of the temperature, is quadratic in the shear rate. Because there is little scale separation in granular flows, there is no compelling reason why continuum descriptions should apply. However, comparisons between predictions of 40 continuum theories and the profiles of concentration, velocity, and temperature measured in physical experiments and discrete numerical simulations of steady flows show relatively good agreement $^{6-9}$ and this encourages their use.

Above a volume fraction of 0.49 , at which a first-order phase 45 transition between disordered and ordered states in an equilibrated system of colliding hard spheres is first possible, ${ }^{10}$ molecular dynamics simulations show that correlations between collisions begin to influence the relations between the components of the stress and the shear rate. ${ }^{11-13}$ The introduction 50 of an additional length scale in the relation for the rate of collisional dissipation of fluctuation energy associated with the size of clusters of interacting spheres modifies the stress relations in an appropriate way. ${ }^{9,14-16}$ The length scale is determined by the competition between the orienting influence of the flow and the ${ }_{55}$ randomizing influence of the collisions, using a local balance between the rates of production and dissipation of fluctuation energy. This approach has been tested against discrete element simulations of steady flows in a variety of flow configurations. ${ }^{17-}$ 20

60 These extended stress relations apply until the mean separation distance between the edges of the spheres vanishes at least along the direction of principal compression, at which point, the stresses for hard spheres become singular. ${ }^{20}$ The volume fraction at which this occurs is less than that for random close packing 65 and is seen in numerical simulation to depend on the coefficient of sliding friction. ${ }^{21}$ It can be interpreted as "the" jamming transition for shearing flows of compliant, frictional spheres. ${ }^{1,22-}$ ${ }^{25}$ However, discrete numerical simulations in two dimensions of the shearing of compliant, frictional, circular disks ${ }^{26-28}$ indicate 70 that there are three different jamming transitions. Consequently, more work is required in three dimensions to determine if, in fact, the volume fraction at which the stresses become singular is unique. We note that, because of the anisotropy inherent in a shearing flow, the critical volume fraction provides only a rough 75 characterization of the singular state; descriptions of the anisotropy of this state are beginning to be developed. ${ }^{29}$

Replacing a rigid contact with a compliant contact has three consequences: it permits the introduction of a time associated 
with the duration of a collision before the hard-sphere singularity $;^{30}$ it relaxes the singularity of the stresses at this singularity; and it allows prediction of the stresses at volume fractions greater than that at the singularity. The stresses at 5 volume fractions beyond the hard-sphere singularity have parts that depend on the shear rate and parts that depend on the deformation of the contact. We refer to these as the ratedependent and rate-independent ${ }^{31,32}$ parts of the stress.

In this paper, we calculate stress relations for steady, shearing 10 flows of deformable, elastic spheres that apply at volume fractions less than, at, and greater than the hard-sphere singularity. We do this by extending the stress relations of kinetic theories to collisions with a finite duration and employing the elastic component of the contact to describe the stresses 15 associated with enduring contact of the spheres.

We first focus on steady, homogeneous shearing flows and compare the predicted stresses with those measured in numerical simulations. $^{21,33,34}$ The predictions compare well over the range of volume fraction before and after the hard sphere singularity and

20 over eleven orders of magnitude of the contact stiffness. The model requires no parameters other than the contact stiffness, coefficient of collisional restitution, and sliding friction of the spheres. An interesting result is that the viscosity below the singularity is seen to increase with the fluctuation energy, as in a 25 dense gas, but to decrease with fluctuation energy above the singularity, as in a liquid. We also consider steady, inhomogeneous shearing flows, in which the conduction of fluctuation energy is important, and reproduce, for spheres, the qualitative feature of stress relations measured in discrete 30 numerical simulations of circular disks. ${ }^{35}$

\section{Theory}

We consider a steady, uniform, unidirectional, shearing flow of spheres of mass density $\rho_{p}$ and diameter $d$. Their interactions are characterized, in part, by a normal coefficient of restitution $e$ and ${ }_{35}$ contact friction $\mu$. We take $v$ to be the solid volume fraction, and $x$ and $y$ to be the flow and shear directions, respectively. Then, $u$ is the only component of the mean velocity of the particles and $u^{\prime}$ is the shear rate, with the prime denoting a derivative with respect to $y$. We define the critical volume fraction, $\mathrm{v}_{c}$, as the volume 40 fraction above which a rate-independent component of the stresses develops. At greater volume fractions, the mean distance between at least some sphere centres is less than one diameter indicating persistent deformations of such particles. ${ }^{24} \mathrm{We}$ distinguish between flows at volume fraction less than and ${ }_{45}$ greater than $\mathrm{v}_{c}$, referring to those at volume fractions less than $v_{c}$ as collisional and those above $\mathrm{v}_{c}$ as deformational. We first carry out calculations for contact forces that are linear in the contact deformation and indicate the modifications for non-linear contacts. Also, for sake of simplicity, we limit our analysis to 50 dense flows; in these, the dependence of the coefficients of kinetic theory on the volume fraction is proportional to that of the radial distribution function at contact.

\section{Collisional regime}

In this regime, the stresses are due to the transfer of momentum 55 in collisions, the mean separation distance between the centres of spheres is greater than one diameter, and the granular material behaves as a dense gas. Consequently, we begin with the stress relations of kinetic theory, extended to include an additional length scale in the rate of collisional dissipation, ${ }^{14,15,18}$ and 60 modify them to incorporate the deformation of a contact during a collision. ${ }^{32}$ When the contact is compliant, the time interval between two successive collisions is equal to the time of free flight, $t_{f}$, plus the duration of the contact, $t_{c}$.

The time of free flight is $\left(\pi^{1 / 2} / 24\right) d /\left(G T^{1 / 2}\right)$, where $G$ is 65 the product of the volume fraction and the radial distribution function at collision and $T$ is the one-third the mean-square of the velocity fluctuations - the granular temperature. ${ }^{36}$ For the volume fraction dependence of the radial distribution function $g_{0}$ of two colliding spheres, we adopt the expression of Vescovi et al., ${ }^{19}$ 70 which smoothly interpolates between the form due to Carnahan and Starling, appropriate at small volume fractions, and that which diverges at a critical volume fraction: ${ }^{20}$

$$
g_{0}=\mathrm{f} \frac{2-\mathrm{v}}{2(1-\mathrm{v})^{3}}+(1-\mathrm{f}) \frac{2}{v_{c}-v},
$$

where $\mathrm{f}=\left(\mathrm{v}_{c}+\mathrm{v}-0.8\right)\left(\mathrm{v}_{c}-\mathrm{v}\right) /\left(\mathrm{v}_{c}-0.4\right)^{2}$. For rigid contacts, 75 shearing ceases at the critical volume fraction and the system is said to be jammed. For deformable contacts, discrete numerical simulations show that the value of the critical volume fraction depends only on the coefficient of friction. ${ }^{21}$

The duration of a collision is proportional to the ratio of the 80 particle diameter to the elastic wave speed in the particle: $c=\left(E / \rho_{p}\right)^{1 / 2}$, where $E$ the Young's modulus of the material of the spheres. ${ }^{30}$ We take the coefficient in the proportion to be $1 / 5$, which provides the best fitting with the simulations of simple shearing shown later. Consequently, the frequency of collisions 85 the inverse of the time interval between two successive collisions - is reduced for deformable particles with respect to that for rigid particles by the factor

$$
\begin{aligned}
\frac{t_{f}}{t_{f}+t_{c}} & =\frac{\pi^{1 / 2}}{24} \frac{d}{G T^{1 / 2}}\left(\frac{\pi^{1 / 2}}{24} \frac{d}{G T^{1 / 2}}+\frac{d}{5 c}\right)^{-1} \\
& =\left[1+\frac{24 G}{5 \pi^{1 / 2}}\left(\frac{\rho_{p} T}{E}\right)^{1 / 2}\right]^{-1},
\end{aligned}
$$

Because the collisional stresses, the collisional rate of dissipation 90 of the fluctuation energy, and the flux of fluctuation energy are all proportional to the frequency of collision, the constitutive relations of extended kinetic theory for rigid particles, a modification of those of Garzo and Dufty, ${ }^{4}$ must be multiplied by the factor of Eq. 1 when applied to deformable spheres.

95 The rigid and deformable contributions to the constitutive relation for the pressure $p$ and the shear stress $s$ are best seen when the inverses of these are written as the sums of the inverses of the two contributions:

$$
p^{-1}=\left[2(1+e) G \rho_{p} v T\right]^{-1}+\left[\frac{5 \pi^{1 / 2} v(1+e)}{12}\left(\rho_{p} E T\right)^{1 / 2}\right]^{-1},
$$




$$
s^{-1}=\left(\frac{8 J G}{5 \pi^{1 / 2}} \rho_{p} v T^{1 / 2} d\left|u^{\prime}\right|\right)^{-1}+\left[\frac{v J}{3}\left(\rho_{p} E\right)^{1 / 2} d\left|u^{\prime}\right|\right]^{-1},
$$

respectively, where the dependence of $J$ on the coefficient of restitution is given by $J=(1+e) / 2+\pi(1+e)^{2}(3 e-1) /\left[96-24(1-e)^{2}-20\left(1-e^{2}\right)\right]$ $5 .{ }^{15}$ The first terms on the right-hand sides of Eqs. 3 and 4 are the inverses of the constitutive relations for rigid spheres; the second terms are the inertial-elastic contributions, ${ }^{37}$ called the intermediate contributions by Chialvo et al., ${ }^{21}$ who explored shearing of deformable spheres in discrete numerical simulations.

${ }_{10}$ Unlike the fits by Chialvo et al. to the constitutive relations, ${ }^{21}$ the expressions in Eqs. 3 and 4 depend also on the granular temperature; this permits the analysis of inhomogeneous shearing flows, such as those between rigid, bumpy boundaries, ${ }^{38}$ in which the temperature is not proportional to the square of the shear rate.

15 A consequence of the extension of the frequency of collisions from rigid to deformable particles is that the pressure and the shear stress are no longer singular at $\mathrm{v}=\mathrm{v}_{c}$. For volume fractions close to the critical, $G$ tends to infinity; hence, the first terms on the right hand side of Eqs. 3 and 4 vanish, while the other terms 20 remain finite.

Similarly, the expressions of the rate of collisional dissipation of the fluctuation energy $\Gamma$ is

$$
\Gamma^{-1}=\left[\frac{12\left(1-\varepsilon^{2}\right) G}{\pi^{1 / 2} L} \rho_{p} v T^{3 / 2}\right]^{-1}+\left[\frac{5\left(1-\varepsilon^{2}\right) \nu}{2 L} 5\left(\rho_{p} E\right)^{1 / 2} T\right]^{-1},
$$

where $\varepsilon$ is an effective coefficient of restitution, which takes into 25 account the additional dissipation of the fluctuation energy due to the rotation and contact friction of the particles. ${ }^{39,40}$ In Eq. 5, $L$ is an additional length scale related to the correlation of collisions at volume fractions larger than $0.49 .{ }^{14}$ It is determined by a balance between the ordering influence of the shearing and the 30 randomizing influence of the collisions:

$$
L=f_{0} d \frac{u^{\prime} d}{T^{1 / 2}},
$$

where discrete numerical simulations indicate that the coefficient $f_{0}$ is singular at random close packing: ${ }^{20}$

$$
f_{0}=\left[\frac{4 J}{15\left(1-\varepsilon^{2}\right)}\right]^{1 / 2}\left[1+\frac{26(1-\varepsilon)}{15} \frac{\max (v-0.49,0)}{0.64-v}\right] .
$$

35 When the flow interacts with boundaries, there is a flux of fluctuation energy directed either into or out of the flow. ${ }^{38,41,42}$ In such situations, the granular temperature must be determined as a solution of the differential equation that expresses the balance of fluctuation energy. The constitutive relation for the inverse of the 40 flux of fluctuation energy $Q$ for deformable spheres is

$$
Q^{-1}=\left(-\frac{4 M G}{\pi^{1 / 2}} \rho_{p} v d T^{1 / 2} T^{\prime}\right)^{-1}+\left[-\frac{5 v M}{6}\left(\rho_{p} E\right)^{1 / 2} d T^{\prime}\right]^{-1} .
$$

where $\quad M=(1+e) / 2+9 \pi(1+e)^{2}(2 e-1) /[128-56(1-e)] .^{15}$ Here, we have ignored the contribution to the energy flux proportional to the gradient of volume fraction, ${ }^{4}$ which is
45 negligible for dense flows.

\section{Deformational regime}

In this case, the mean separation distance, at least along the direction of the principal compression axis, is zero. Volume fractions larger than $v_{c}$ can be achieved in a disordered assembly ${ }_{50}$ of particles only if some particles deform. Therefore, an elastic component of the stresses associated with such persistent deformations develops. For isotropic compression of an isotropic aggregate, the elastic contribution to the pressure is proportional to the product of the normal component of the contact force $P$, 55 the separation between the centres $d$, the number of contacts per sphere $Z$, and the number of spheres per unit volume $6 \mathrm{v} /\left(\pi d^{3}\right)$ .43

$$
p_{e}=\frac{P v Z}{\pi d^{2}}
$$

For linear contacts, $P$ is related to the normal component of the ${ }_{60}$ contact displacement $\delta$ through the contact stiffness $\pi d E / 4:{ }^{33}$

$$
P=\frac{\pi}{4} d E \delta
$$

The contact displacement can be related to the volume fraction at fixed coordination number by treating the particle deformations as overlapping. Then, the ratio of the total overlapped volume to ${ }_{65}$ the particle volume gives the increase in the volume fraction from the critical value,

$$
\frac{v}{v_{c}}=1+\frac{Z 2 \pi \delta^{2}(d / 2-\delta / 3)}{\pi d^{3} / 6} \simeq 1+6 Z\left(\frac{\delta}{d}\right)^{2}
$$

in which that the ratio $\delta / d$ is small. Then, with Eq. $10, p_{e}$ is proportional to the square-root of the product of $\left(\mathrm{v}-\mathrm{v}_{c}\right)$ and $Z$. 70 Discrete numerical simulations of simple shearing of frictional, deformable spheres $^{33}$ indicate that the coordination number is approximately linear in the increase of volume fraction from $\mathrm{v}_{c}$, with a slope of about ten, at least for the softest particles. With this, we find that the elastic pressure is roughly equal to the 75 product of $E$ and the excess of the volume fraction above the critical. On the basis of fitting with numerical simulations, we adopt the expression

$$
p_{e}=0.6 \frac{\pi}{4}\left(v-v_{c}\right) E
$$

For stiffer particles, the coordination number scales with the 80 square root of the increase of volume fraction from $v_{c},{ }^{29,33}$ but the pressure still increases linearly with the difference $v-v_{c}$ in steady, homogeneous shearing. ${ }^{29}$ Consequently, we assume that Eq. 12 holds for any value of the particle stiffness.

The shearing forces individual particles to make and break ${ }_{85}$ chains of frictional contacts, sometimes violently; ${ }^{44}$ so that a component of the stresses associated with the transfer of momentum is still present at $v>v_{c}$, with the frequency of transfer equal to the inverse of the contact duration. We model this elastic-inertial pressure in the same way as we model the inertial90 elastic pressure in the collisional regime, taking $v=v_{c}$. The total pressure is the sum of the elastic-inertial and the purely elastic 
components,

$$
p=\frac{5 \pi^{1 / 2} v_{c}(1+e)}{12}\left(\rho_{p} E T\right)^{1 / 2}+0.6 \frac{\pi}{4}\left(v-v_{c}\right) E .
$$

The first and second term on the right hand side of Eq. 13 can be identified with, respectively, the intermediate and quasi-static 5 pressures of Chialvo et al. ${ }^{21}$

We take the constitutive relation for the elastic-inertial shear stress to be the inverse of the inertial-elastic contribution to Eq. 4 with $v=v_{c}$. Once again, the total shear stress is the sum of two components: the elastic-inertial and the purely elastic. In the

10 steady state, both the purely elastic shear stress and the purely elastic pressure $p_{e}$ are proportional to the stiffness, so they are proportional to each other. Hence, the total shear stress may be written as

$$
s=\frac{v_{c} J}{3}\left(\rho_{p} E\right)^{1 / 2} d\left|u^{\prime}\right|+\eta p_{e} .
$$

15 where $\eta$ is the constant of proportionality. Discrete numerical simulations of unsteady, homogeneous shearing in the rateindependent regime, ${ }^{29}$ indicate that the ratio of the purely elastic shear stress to the purely elastic pressure is proportional to the anisotropy of the contact network. Because the ratio of the 20 elastic-inertial shear stress to the elastic-inertial pressure is sensitive to this anisotropy in the same way, we determine $\eta$ from this ratio:

$$
\eta=\frac{4 J}{5 \pi^{1 / 2}(1+e)} \frac{d\left|u^{\prime}\right|}{T^{1 / 2}} .
$$

The values of $\eta$ obtained from Eq. 15 in simple shearing ${ }^{20}$ with 25 different particle friction coefficients are in agreement with the measurements of the constant stress ratio obtained in numerical simulations when $v>v_{c}$ and the particles are sufficiently rigid. ${ }^{21}$

Because of the energy released by the breaking of chains, the rate of dissipation of the fluctuation energy and the fluctuation 30 energy flux also persist at volume fractions larger than the critical. We take the dissipation rate to be the inverse of the inertial-elastic contribution to Eq. 5 with $v=v_{c}$,

$$
\Gamma=\frac{5\left(1-\varepsilon^{2}\right) v_{c}}{2 L}\left(\rho_{p} E\right)^{1 / 2} T,
$$

and we assume that the correlation length is still given by Eq. 6, 35 with $f_{0}$ evaluated at $v=v_{c}$. Finally, we take the flux of fluctuation energy to be the inverse of the inertial-elastic contribution to Eq. 8 with $v=v_{c}$,

$$
Q=-\frac{5 v_{c} M}{6}\left(\rho_{p} E\right)^{1 / 2} d T^{\prime} .
$$

\section{Modifications for Hertzian contacts}

40 Real spheres interact through Hertzian contacts. In this case, the duration of contact is ${ }^{45}$

$$
t_{c}=1.47\left(\frac{5 \sqrt{\pi}}{4} \rho_{p} \frac{1-\psi^{2}}{E}\right)^{2 / 5} \frac{d}{(T / 3)^{1 / 10}},
$$

where $\psi$ is Poisson's ratio, and we have assumed the precollisional velocity equal to $(T / 3)^{1 / 2}$. Then, Eq. 2 becomes

$$
45 \quad \frac{t_{f}}{t_{f}+t_{c}}=\left[1+1.47 \frac{24 G}{\pi^{1 / 2}}\left(\frac{3^{1 / 4} 5 \sqrt{\pi}}{4} \rho_{p} \frac{1-\psi^{2}}{E} T\right)^{2 / 5}\right]^{-1} \text {. }
$$

Using this, the derivation the constitutive relations for the inertial stresses, the rate of dissipation and the flux of fluctuation energy is straightforward.

For Hertzian spheres, the normal contact force is ${ }^{43}$

$$
P=\frac{2}{9 \sqrt{3}} \frac{S d^{2}}{1-\psi}\left(\frac{6 \delta}{d}\right)^{3 / 2},
$$

where $S$ is the shear modulus. Equation 20 must be used instead of Eq. 10 in Eq. 9 to calculate the purely elastic pressure. If the relation between the volume fraction and the coordination number is approximately linear, we obtain

55

$$
p_{e} \propto \frac{S}{1-\psi}\left(v-v_{c}\right)
$$

where the coefficient of proportionality is of order one. This dependence has been observed in the numerical simulations. ${ }^{21}$

\section{Simple shearing}

We next test the theory against numerical simulations of steady, ${ }_{60}$ homogeneous shearing of compliant, frictional spheres that interact through linear springs and dashpots in parallel. ${ }^{21,33,34}$ In this case, Young's modulus is $E=4 k /(\pi d)$, where $k$ is the stiffness of the normal spring. ${ }^{33}$

In simple shearing, the volume fraction and the granular 65 temperature are uniform, so that the balance of fluctuation energy reduces to

$$
\left(s-\eta p_{e}\right)\left|u^{\prime}\right|=\Gamma,
$$

where we have assumed that the work of the purely elastic shear stress is completely recoverable and does not produce fluctuation 70 energy. ${ }^{31,32}$ This gives

$$
\frac{T}{u^{\prime 2} d^{2}}=\frac{2 J}{15\left(1-\varepsilon^{2}\right)}\left[1+\frac{26(1-\varepsilon)}{15} \frac{v-0.49}{0.64-v}\right],
$$

for volume fractions less than the critical, and

$$
\frac{T}{u^{\prime 2} d^{2}}=\frac{2 J}{15\left(1-\varepsilon^{2}\right)}\left[1+\frac{26(1-\varepsilon)}{15} \frac{v_{c}-0.49}{0.64-v_{c}}\right],
$$

for volume fractions larger than the critical. Equations 23 and 24 75 imply that in simple shearing, the granular temperature, made dimensionless with the product of the particle diameter and the shear rate, is a function only of the volume fraction, and constant for $v>v_{c}$.

In Fig. 1a and 1b, we compare the predicted dimensionless ${ }_{80}$ granular temperature with the measurements in discrete numerical simulations of simple shearing: in Fig. 1a, when 


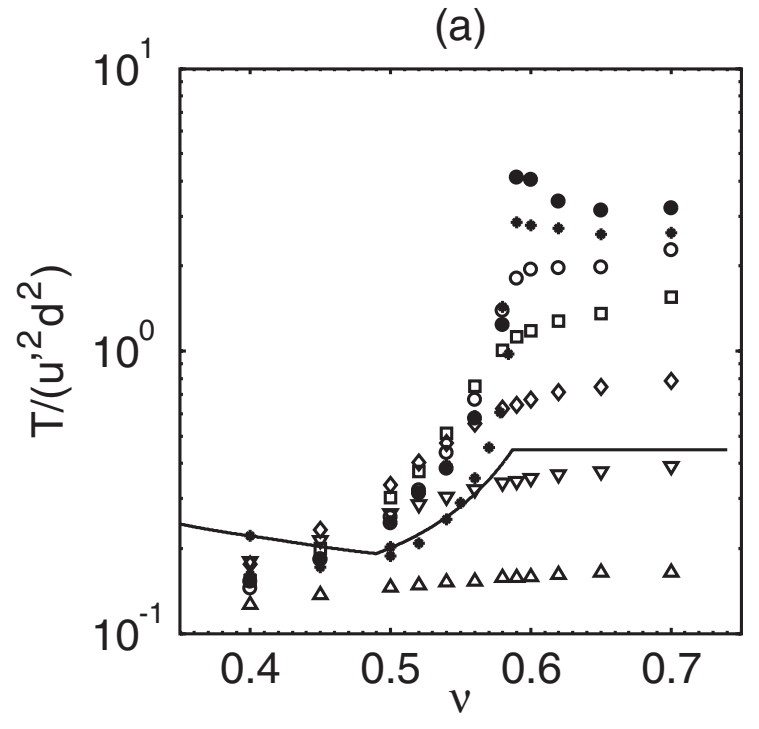

(b)

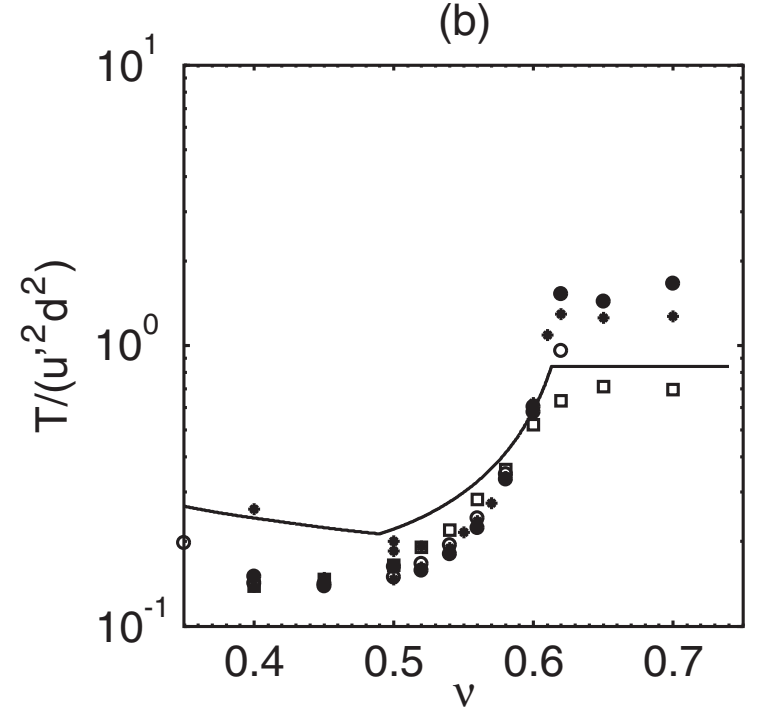

Fig. 1 Dimensionless granular temperature as a function of the volume fraction: predicted (lines) and measured by Ji and $\mathrm{Shen}^{33}$ and Chialvo and 5 Sundaresan $^{34}$ (symbols). Here, and in the following plots, the measured results are for dimensionless particle stiffness equal to: $10^{1}$ (hollow upper triangles); $10^{2}$ (hollow lower triangles); $10^{3}$ (hollow diamonds); $10^{4}$ (hollow squares); $10^{5}$ (hollow circles); $10^{6}$ (stars); $10^{7}$ (solid circles); $10^{8}$ (solid squares); $10^{9}$ (solid diamonds); $10^{10}$ (solid lower triangles); $10^{11}$

$e=0.7$ and $\mu=0.5$, so that $v_{c}=0.587$ and $\varepsilon=0.53^{21,40}$; and, in Fig. $1 b$, when $e=0.7$ and $\mu=0.1$, so that $v_{c}=0.613$ and $\varepsilon=0.59 .^{21,40}$ The numerical simulations have been performed for dimensionless particle stiffness $k /\left(\rho_{p} u^{2} d^{3}\right)$ ranging from 10 to $1510^{7}$.

The agreement is good when $\mu=0.1$, and acceptable when $\mu=0.5$, at least for volume fractions less than the critical value. As anticipated, the dimensionless granular temperature is nearly constant beyond the transition, although its value increases with 20 the dimensionless particle stiffness and seems to saturate, if the spheres are stiff enough. We associate the deviations from the numerical simulations with multiple interactions, which are not presently incorporated in the inertial parts of the constitutive relations.

25 We could certainly improve the agreement with the measurements by modifying the function $f_{0}$ in the correlation length, which has been accurately tested only against the results of numerical simulations of frictionless particles, ${ }^{19}$ to incorporate the influence of the particle friction, and by modelling the role of 30 multiple interactions, at least in the dissipation rate. We prefer to avoid those additional complications for several reasons. First, the agreement between the theory and the numerical results is less adequate when $\mu=0.5, v>v_{c}$ and the particles are stiff. In that case, the elastic-inertial component is a small fraction of the total 35 stress, so that a poor estimate of the granular temperature does not substantially affect the predictions of the pressure and the shear stress. Also, a friction coefficient of 0.5 is rather large; most real particles are characterized by friction coefficients closer to $0.1{ }^{46}$ Finally, the measurements of $\mathrm{Ji}$ and $\mathrm{Shen}^{33}$ differ from 40 those of Chialvo and Sundaresan ${ }^{34}$ for the same dimensionless stiffness of $10^{6}$, indicating an uncertainty in the measurements. We postpone the refinement of the model to improve the prediction of the granular temperature for large friction and to include the role of multiple collisions to future work.

${ }_{45}$ We can re-write the full constitutive relations for the pressure and the shear stress in dimensionless terms using the density and diameter of the spheres, the shear rate, and the particle stiffness instead of Young's modulus, making use of Eq. 15:

$$
\begin{aligned}
\left(\frac{p}{\rho_{p} u^{\prime 2} d^{2}}\right)^{-1} & =\left[2(1+e) v G \frac{T}{u^{\prime 2} d^{2}}\right]^{-1} \\
& +\left[\frac{5 \pi^{1 / 2} v(1+e)}{12}\left(\frac{4}{\pi} \frac{T}{u^{\prime 2} d^{2}} \frac{k}{\rho_{p} u^{\prime 2} d^{3}}\right)^{1 / 2}\right]^{-1},
\end{aligned}
$$

50 if $\mathrm{V}<\mathrm{v}_{c}$, while

$$
\begin{aligned}
\frac{p}{\rho_{p} u^{\prime 2} d^{2}}= & \frac{5 \pi^{1 / 2} v_{c}(1+e)}{12}\left(\frac{4}{\pi} \frac{T}{u^{\prime 2} d^{2}} \frac{k}{\rho_{p} u^{\prime 2} d^{3}}\right)^{1 / 2} \\
& +0.6\left(v-v_{c}\right) \frac{k}{\rho_{p} u^{\prime 2} d^{3}},
\end{aligned}
$$

if $\mathrm{v} \geq \mathrm{v}_{c}$; and

$$
\begin{aligned}
\left(\frac{s}{\rho_{p} u^{\prime 2} d^{2}}\right)^{-1}= & {\left[\frac{8 J v G}{5 \pi^{1 / 2}}\left(\frac{T}{u^{\prime 2} d^{2}}\right)^{1 / 2}\right]^{-1} } \\
& +\left[\frac{v J}{3}\left(\frac{4}{\pi} \frac{k}{\rho_{p} u^{\prime 2} d^{3}}\right)^{1 / 2}\right]^{-1},
\end{aligned}
$$

if $\mathrm{v}<\mathrm{v}_{c}$, while

$$
\frac{s}{\rho_{p} u^{\prime 2} d^{2}}=\frac{4 J}{5 \pi^{1 / 2}(1+e)}\left(\frac{T}{u^{\prime 2} d^{2}}\right)^{-1 / 2} \frac{p}{\rho_{p} u^{\prime 2} d^{2}},
$$

if $v \geq v_{c}$. In simple shearing, these are functions only of $v$, through Eqs. 23 and 24, and the dimensionless stiffness.

Figures $2 \mathrm{a}$ and $2 \mathrm{~b}$ show the predicted dimensionless pressure and shear stress, compared with the results of numerical 


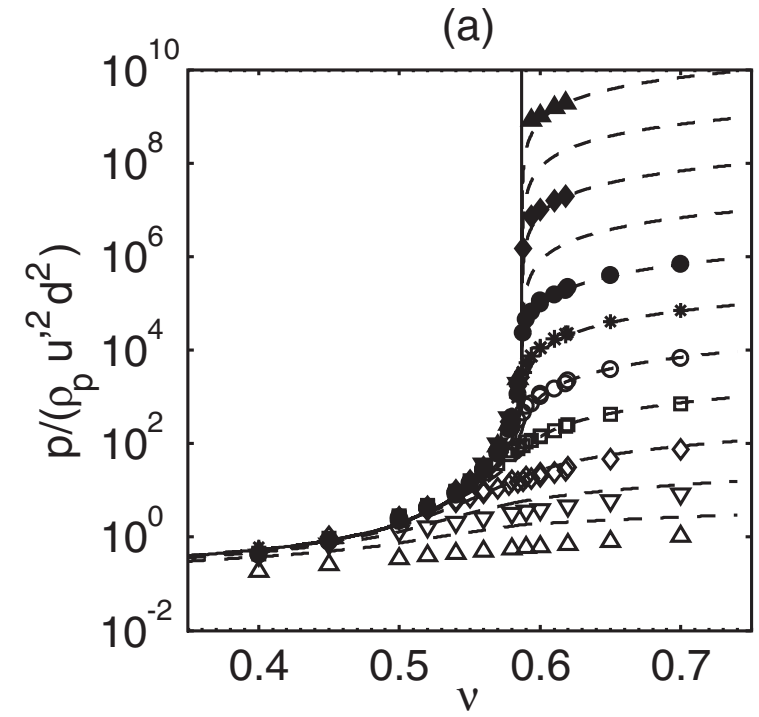

(b)

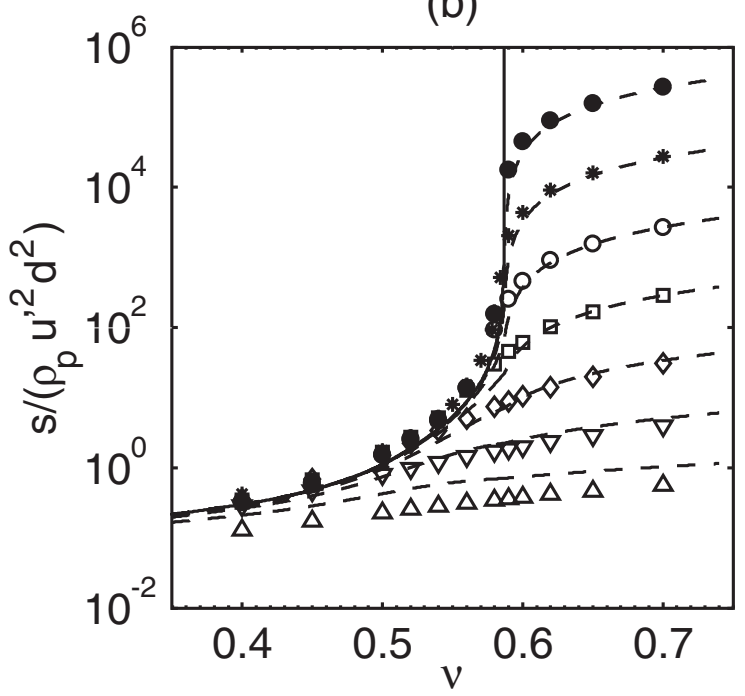

Fig. 2 Dimensionless (a) pressure and (b) shear stress as functions of the volume fraction as predicted (dotted lines) and measured by Ji and Shen, ${ }^{33}$ 5 Chialvo et al. ${ }^{21}$ and Chialvo and Sundaresan ${ }^{34}$ (symbols), when $e=0.7$ and $\mu=0.5$. The symbols have the same significance as in Fig. 1. Also shown are the theoretical curves for perfectly rigid particles (solid lines).

simulations of simple shearing, ${ }^{21,33,34}$ for a range of dimensionless stiffness, $k /\left(\rho_{p} u^{2} d^{3}\right)$, from 10 to $10^{11}$ when $e=0.7$ and $\mu=0.5$.

${ }_{10}$ Also shown are the curves for rigid particles, that is, for infinite $k$, for which the collisions are instantaneous and binary and no disordered assembly is possible beyond $v_{c}$. The predictions agree well with the simulations. The volume fraction at which the data depart from the rigid curve increases with the particle stiffness.

${ }_{15}$ For real spheres, the collisions can be considered instantaneous and binary up to volume fractions close to the critical value.

If the stiffness and the particle diameter are used to scale the stresses, the results from the numerical simulations collapse at volume fractions larger than $\mathrm{v}_{c}$, where the purely elastic stresses 20 dominate, as already observed by Ji and Shen. ${ }^{33}$ The agreement between the predictions and the numerical simulations is again remarkable (Fig. 3).

Figure 4 shows the comparison between the present theory, the (a)

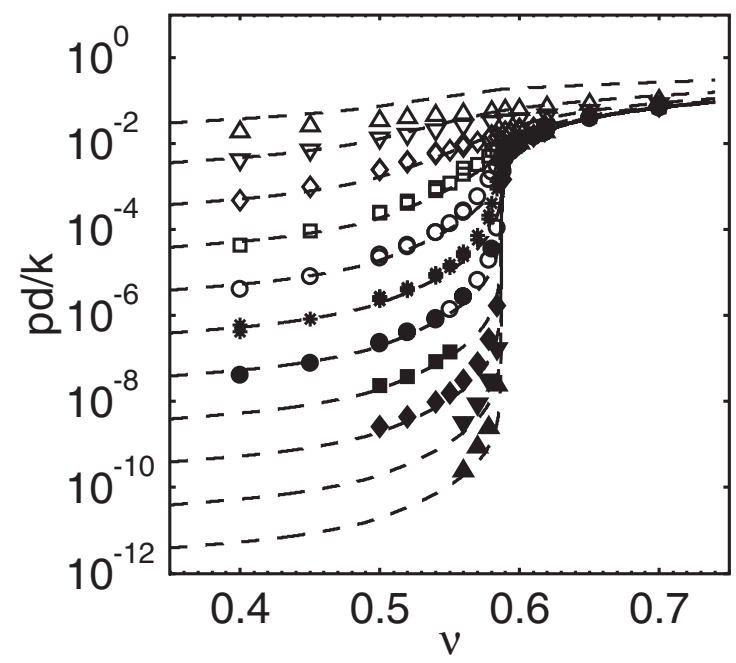

(b)

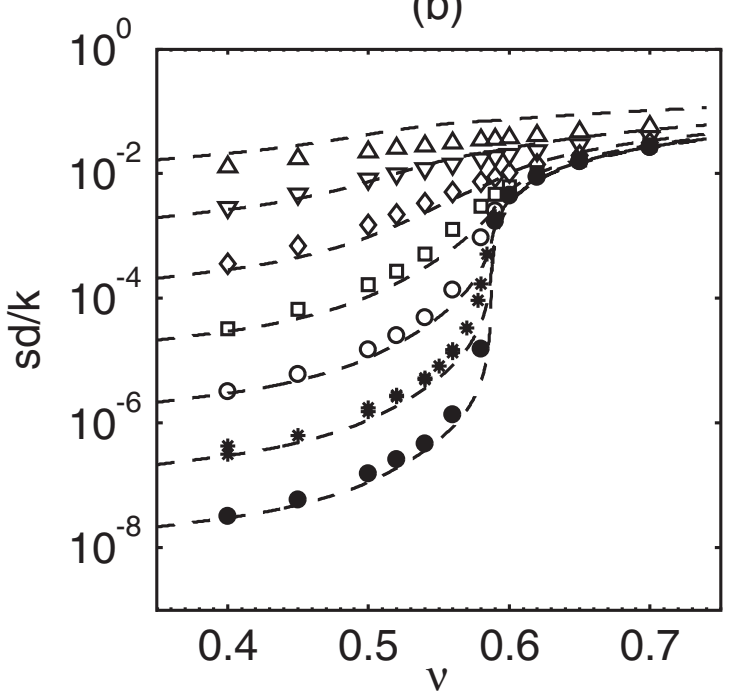

Fig. 3 (a) Pressure and (b) shear stress made dimensionless using the particle stiffness and diameter versus volume fraction as predicted (dotted lines) and measured by Ji and Shen, ${ }^{33}$ Chialvo et al.,${ }^{21}$ and Chialvo and Sundaresan $^{34}$ (symbols) when $e=0.7$ and $\mu=0.5$. The symbols have the 30 same significance as in Fig. 1.

constitutive relation for the pressure suggested by Chialvo et al. ${ }^{21}$ through fitting with their simulations, and the measurements in discrete numerical simulations ${ }^{21,33,34}$ when $e=0.7, \mu=0.5$, and the dimensionless stiffness is $10^{6}$. The data tests the validity of 35 the linear dependence of the purely elastic pressure on the volume fraction against the power law dependence with an exponent equal to $2 / 3$ suggested by Chialvo et al. ${ }^{21}$

Figure 5 shows the comparison between the predicted dimensionless pressure and shear stress and those measurements 40 in discrete numerical simulations by Chialvo et al., ${ }^{21}$ Chialvo and Sundaresan, ${ }^{34}$ and Shen (personal communication) for various dimensionless stiffness, when $e=0.7$ and $\mu=0.1$. 


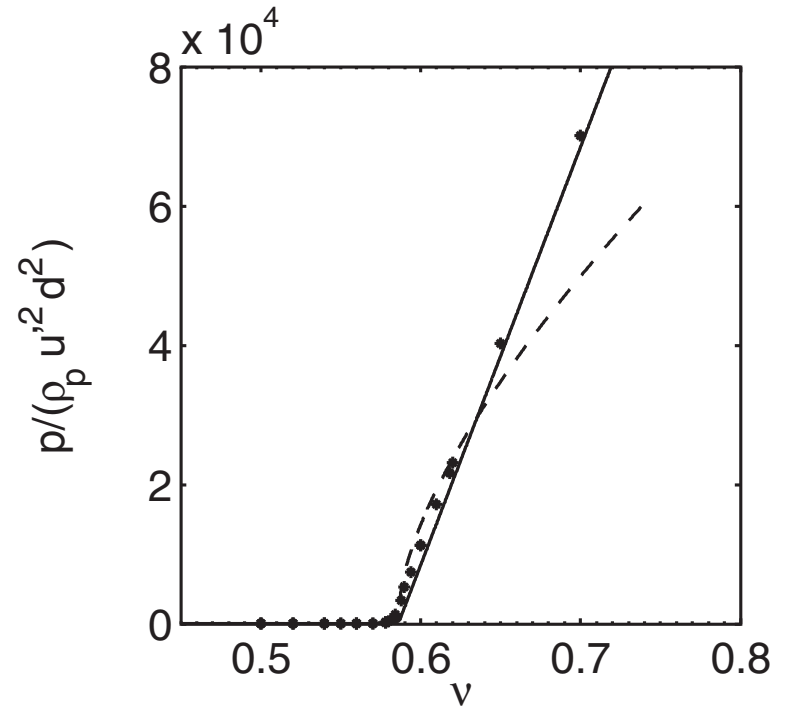

Fig. 4 Dimensionless pressure as a function of the volume fraction as predicted by the present model (solid line), the model of Chialvo et al. ${ }^{21}$ (dotted line) and measured by Ji and Shen,${ }^{33}$ Chialvo et al. ${ }^{21}$ and Chialvo 5 and Sundaresan ${ }^{34}$ (symbols) when $e=0.7, \mu=0.5$ and $k /\left(\rho_{p} u^{2} d^{3}\right)=10^{6}$.

\section{Inhomogeneous shearing in the deformational regime}

In inhomogeneous, steady flows, in which $v>v_{c}$, the shear rate is small and the production of fluctuation energy is negligible. In 10 this case, that the balance of fluctuation energy is, simply,

$$
-\frac{\mathrm{d} Q}{\mathrm{~d} y}=\Gamma
$$

where $y$ is taken to increase in the direction of increase of the volume fraction. That is, the diffusion of the fluctuation energy balances its dissipation. ${ }^{42}$ Using Eqs. 16 and 17, we obtain

$$
\frac{\mathrm{d}^{2} T}{\mathrm{~d} y^{2}}=\frac{1}{\lambda^{2}} T,
$$

where $\lambda^{2}=d L M /\left[3\left(1-\varepsilon^{2}\right)\right]$. Equation 28 can be solved to determine the distribution of the granular temperature in the flow. As a first approximation, we take the correlation length $L$ to be constant and equal to its value in simple shearing, determined 20 using Eq. 24 in Eq. 6,

$$
\frac{L}{d}=1+\frac{26(1-\varepsilon)}{15} \frac{v_{c}-0.49}{0.64-v_{c}} .
$$

With this, $\lambda$ is constant and the solution to Eq. 28 is

$$
T \propto \exp \left(-\frac{y}{\lambda}\right)
$$

(a)

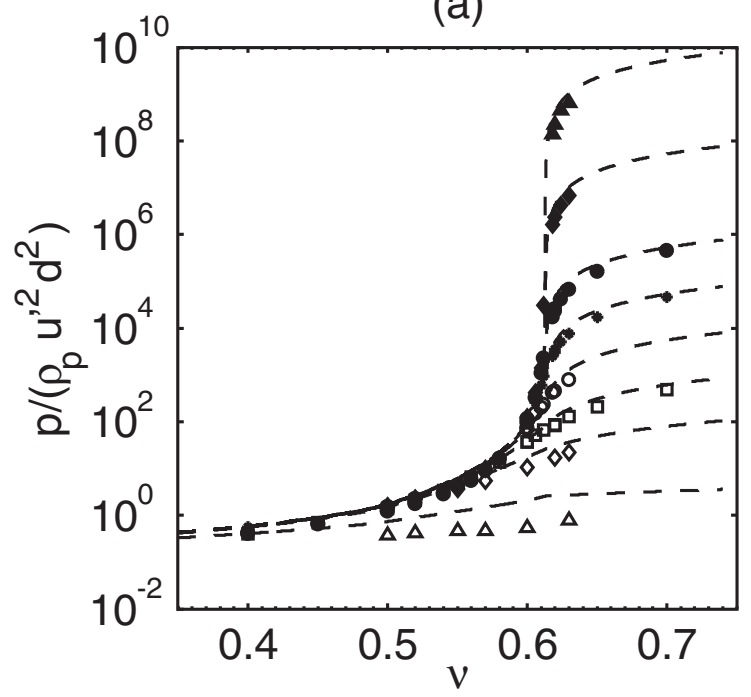

(b)

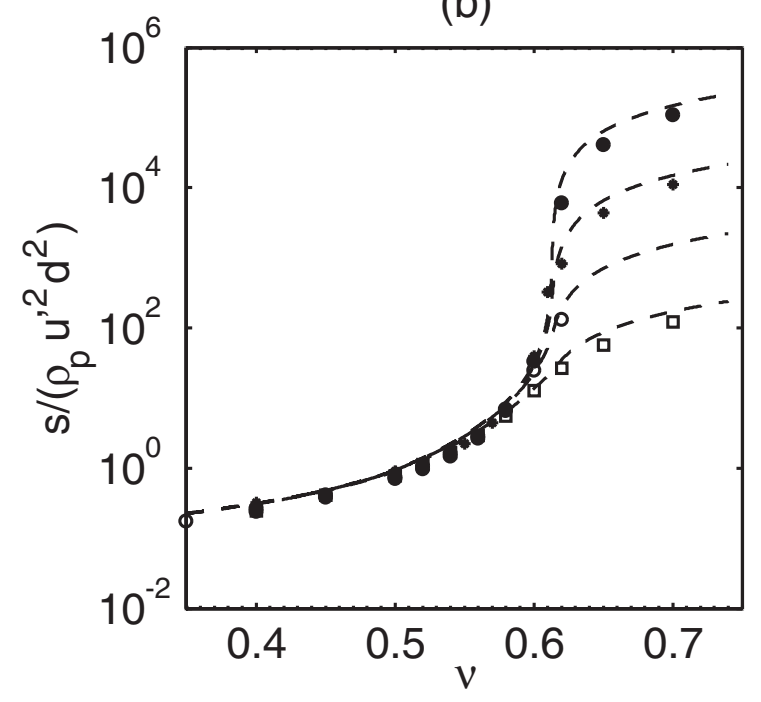

Fig. 5 Dimensionless pressure (a) and shear stress (b) as functions of the volume fraction as predicted (dotted lines) and measured by Chialvo et al. ${ }^{21}$ Chialvo and Sundaresan, ${ }^{34}$ and Shen (personal communication) (symbols) when $e=0.7$ and $\mu=0.1$. The symbols have the same significance as in Fig. 1.

\section{Creeping flow}

Given the distribution of temperature, we seek to determine the velocity profile in the creeping bed below an inclined, collisional shearing flow that is driven by gravity. ${ }^{47}$ There the stress ratio is 35 given by Eq. 15, and is proportional to the ratio of the shear rate 
(a)

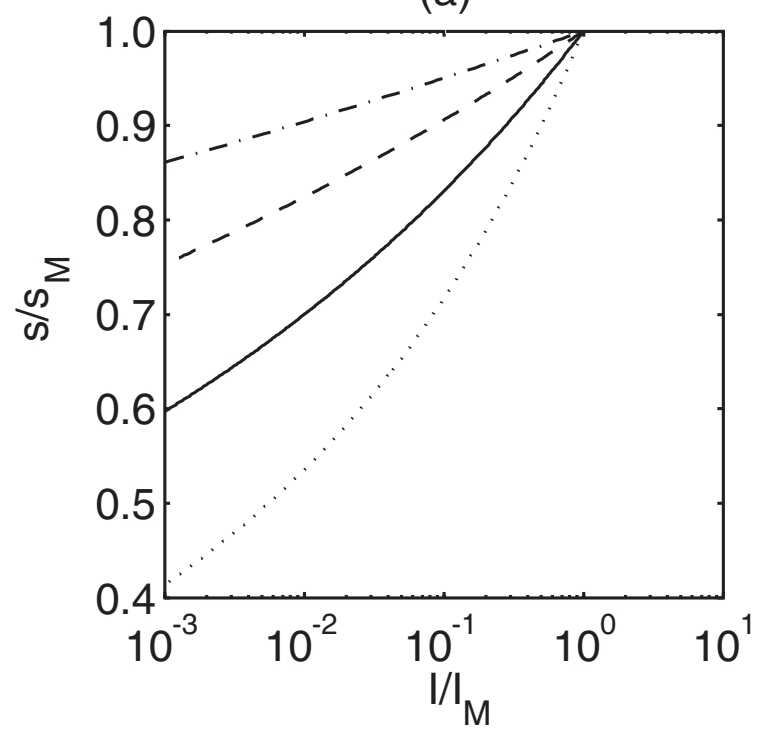

(b)

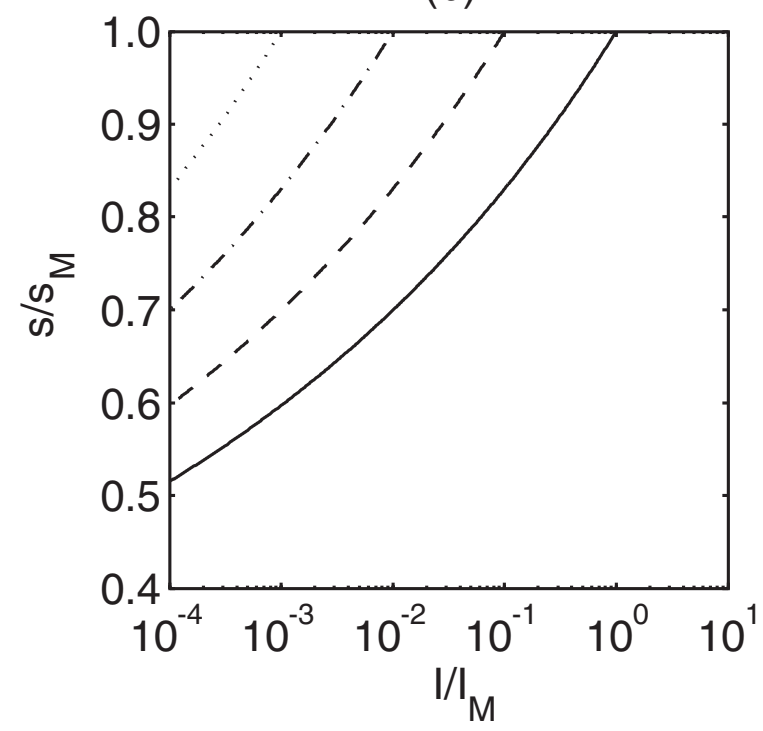

Fig. 6 Normalized stress ratio versus normalized inertial parameter in an annular shear cell for $e=0.7$ and $\mu=0.5$ and: (a) $U_{R} / U_{M}=1$ and $R=25$

5 (dotted line), $R=50$ (solid line), $R=100$ (dashed line) and $R=200$ (dotdashed line); (b) $R=50$ and $U_{R} / U_{M}=1$ (solid line), $U_{R} / U_{M}=0.1$ (dashed line), $U_{R} / U_{M}=0.01$ (dot-dashed line) and $U_{R} / U_{M}=0.001$ (dotted line).

to the square root of the granular temperature. If there are no sidewalls in such a flow, the ratio of the shear stress to the 10 pressure is constant and equal to the tangent of the angle of inclination of the free surface. Then, the shear rate in the bed is directly proportional to the square root of the granular temperature and the velocity decays exponentially, with a decay length equal to $2 \lambda$. For $e=0.7$ and $\mu=0.5$, the decay length of 15 the velocity is roughly equal to two diameters, close to what is observed in the creeping flow of glass spheres. ${ }^{47}$

\section{Annular shear flow}

Another interesting flow is annular shearing. This was investigated by Koval et al. ${ }^{35}$ in two-dimensional discrete element 20 simulations of compliant, frictional, circular disks and modelled by Kamrin and $\mathrm{Koval}^{48}$ using a second-order differential equations for the shear rate. Here, we focus on the threedimensional case, in which spheres are sheared between two coaxial cylinders, with the gravity acting perpendicular to the ${ }_{25}$ plane of shearing. We solve for the flow field in a plane perpendicular to the axis of the cylinders. In doing this, we take $R$ to be the radius of the inner cylinder, which is rotating at constant angular velocity, and $2 R$ to be the radius of the outer cylinder, which is fixed, as in Koval et al. ${ }^{35}$ In this case, $y$ is the radial 30 coordinate, with $y=0$ at the centre of the cylinders. The shear stress in the flow is distributed according to

$$
s=s_{R}\left(\frac{R}{y}\right)^{2},
$$

where $s_{R}$ is the value of the shear stress at the surface of the inner cylinder; while the pressure $p$ is essentially constant. ${ }^{35} \mathrm{We}$ 35 assume that the volume fraction is everywhere larger than $\mathrm{v}_{c}$. This is the case when the tangential velocity of the inner cylinder $U_{R}$ is less than a certain value $U_{M} \cdot{ }^{35}$ The numerical simulations indicate that $s_{R}$ is independent of the angular velocity of the inner cylinder, so $s_{R}=s_{M}$, and the ratio $s_{M} / p$ is equal to the value $\eta$ in ${ }_{40}$ simple shearing. ${ }^{35}$ With the stress ratio given by Eq. 15 , and Eq. 30, we obtain

$$
I=\frac{1}{\left(p / \rho_{p}\right)^{1 / 2}} \frac{5 \pi^{1 / 2}(1+e)}{4 J} \frac{s_{M}}{p}\left(\frac{R}{y}\right)^{2} T_{R}^{1 / 2} \exp \left(-\frac{y-R}{2 \lambda}\right),
$$

where $I=d\left|u^{\prime}\right| /\left(p / \rho_{p}\right)^{1 / 2}$ is the so called inertial parameter. ${ }^{49}$

We assume that the slip velocity of the particles at the surface 45 of the inner cylinder is proportional to the square root of the granular temperature, as is the case for collisional flows over bumpy walls. ${ }^{41}$ Then, $U_{R} / U_{M}=\left(T_{R} / T_{M}\right)^{1 / 2}$ and Eq. 32 can be written as

$$
\frac{I}{I_{M}}=\frac{U_{R}}{U_{M}}\left(\frac{R}{y}\right)^{2} \exp \left(-\frac{y-R}{2 \lambda}\right)
$$

50 where

$$
I_{M}=\frac{5 \pi^{1 / 2}(1+e)}{4 J} \frac{s_{M}}{p} \frac{T_{M}^{1 / 2}}{\left(p / \rho_{p}\right)^{1 / 2}}
$$

is the value of the inertial parameter at the surface of the inner cylinder when $U_{R}=U_{M}$. When $U_{R}=U_{M}$, the granular temperature at $y=R$ is $T_{M}$, and, from Eq. 13, has the value

$$
T_{M}=\frac{144 p^{2}}{25 \pi v_{c}^{2}(1+e)^{2} \rho_{p} E}
$$

If the value of $R$ is changed at constant angular velocity, with $U_{R}=U_{M}, e=0.7$ and $\mu=0.5$, we may employ Eqs. 31 and 33 to obtain the curves shown in Fig. 6a. If $R$ is kept constant and the ratio $U_{R} / U_{M}$ is changed at constant pressure, we obtain the curves 60 of Fig. $6 \mathrm{~b}$. The quantities $s / s_{M}$ and $I / I_{M}$ are the shear stress and the inertial parameter, normalized by their respective values at the inner cylinder. These are in qualitative agreement with the results of the numerical simulations of Koval et al., ${ }^{35}$ shown in 
their Figures $8 \mathrm{~b}$ and 10 . In both cases, the ratio $s / s_{M}$, is less than unity; that is, the ratio of the shear stress to the pressure is less than the yield stress ratio in shear.

\section{Conclusions}

${ }_{5}$ We have proposed constitutive relations for the steady, shearing flows of deformable, inelastic spheres that extend kinetic theories of granular gases to incorporate correlated, non-instantaneous collisions and rate-independent, elastic stresses. The latter are associated with the development of persistent deformations of the 10 particles at volume fractions larger than a critical. The deformability of the particles plays a crucial role in the model. It permits a smooth transition between the collisional and deformational regimes and results in stress components in the deformational regime that contain both static contributions from

15 particle elasticity and dynamic contributions from the momentum transfer associated with the release of elastic energy by the breaking of force chains.

The theory has been tested against previous numerical simulations of steady, homogeneous shearing of inelastic, 20 frictional spheres that interact through linear elastic contacts with stiffnesses that range over eleven orders of magnitude. The theory also has the capacity to reproduce the exponentially decaying velocity profile in erodible beds and the dependence of the velocity on the radius and the angular velocity of the inner 25 cylinder in annular shear flows. Although the constitutive relations have been developed for dry granular flows with inertial and frictional-elastic interactions, they are also relevant to the description of shear thickening in dense suspensions. ${ }^{50,51}$ In such systems, a rapid increase in viscosity accompanies the jamming 30 of frictional particles. For the slow shearing of compliant, frictional spheres, we anticipate that constitutive relations similar to those derived here, with the inertial interactions replaced by viscous interactions at volume fractions less than the critical, will predict the increases in viscosity seen in the numerical 35 simulations and physical experiments. ${ }^{52}$

A final remark concerns the dependence of the viscosity - ratio of the shear stress to the shear rate - on the granular temperature predicted by the theory. When shearing takes place at a volume fraction below the critical, Eq. 4 shows that the viscosity is 40 proportional to $T^{1 / 2}$; that is, it increases with the granular temperature. This is the dependence of the molecular viscosity on the thermodynamic temperature seen in molecular gases. On the contrary, when shearing takes place at a volume fraction above the critical, Eqs. 13 through 15 show that the viscosity is 45 inversely proportional to $T^{1 / 2}$; that is, it decreases with the granular temperature. This is the dependence of the molecular viscosity on the thermodynamic temperature seen in liquids. Interestingly, statistical physics models for the viscosity in liquids introduce a potential energy to predict the decrease of the 50 viscosity with the temperature.$^{53}$ Here, we have obtained a similar result by means of mechanical arguments. Therefore, at least with respect to the dependence of the viscosity on the temperature, the critical volume fraction distinguishes between granular gases and granular liquids.

\section{${ }_{55}$ Notes and references}

${ }^{a}$ Department of Civil and Environmental Engineering, Politecnico di Milano, 20133 Milano, Italy.E-mail: diego.berzi@polimi.it

${ }^{b}$ School of Civil and Environmental Engineering, Cornell University, Ithaca, NY 14853, USA.E-mail: jtj2@cornell.edu

60 1. E. Irani, P. Chaudhuri, and C. Heussinger, Phys. Rev. Lett., 2014, 112, 188303.

2. R. Piazza, Rep. Prog. Phys., 2014, 77, 056602.

3. J. Jenkins and S. Savage, J. Fluid Mech., 1983, 130, 187-202.

4. V. Garzó and J. W. Dufty, Phys. Rev. E, 1999, 59, 5895-5911.

65 5. I. Goldhirsch, Annu. Rev. Fluid Mech., 2003, 35, 267-293.

6. E. Azanza, F. Chevoir, and P. Moucheront, J. Fluid Mech., 1999, 400, 199-227.

7. Y. Forterre and O. Pouliquen, Phys. Rev. Lett., 2001, 86, 5886.

8. H. Xu, M. Louge, and a. Reeves, Contin. Mech. Thermodyn., 2003, $70 \quad \mathbf{1 5}, 321-349$.

9. J. T. Jenkins, Phys. Fluids, 2006, 18, 103307.

10. S. Torquato, Phys. Rev. E, 1995, 51, 3170-3182.

11. N. Mitarai and H. Nakanishi, Phys. Rev. Lett., 2005, 94, 128001.

12. N. Mitarai and H. Nakanishi, Phys. Rev. E, 2007, 75, 031305.

75 13. V. Kumaran, J. Fluid Mech., 2009, 632, 145-198.

14. J. T. Jenkins, Granul. Matter, 2007, 10, 47-52.

15. J. T. Jenkins and D. Berzi, Granul. Matter, 2010, 12, 151-158.

16. D. Berzi, Acta Mech., 2014, 225, 2191-2198.

17. D. Berzi and J. T. Jenkins, Phys. Fluids, 2011, 23, 013303.

80 18. J. T. Jenkins and D. Berzi, Granul. Matter, 2012, 14, 79-84.

19. D. Vescovi, D. Berzi, P. Richard, and N. Brodu, Phys. Fluids, 2014, 26, 053305 .

20. D. Berzi and D. Vescovi, Phys. Fluids, 2015, 27, 013302.

21. S. Chialvo, J. Sun, and S. Sundaresan, Phys. Rev. E, 2012, 85, 021305.

22. A. J. Liu and S. R. Nagel, Annu. Rev. Condens. Matter Phys., 2010, 1, 347-369.

23. C. Heussinger, P. Chaudhuri, and J.-L. Barrat, Soft Matter, 2010, 6, 3050-3058.

90 24. M. van Hecke, J. Phys. Condens. Matter, 2010, 22, 033101.

25. L. E. Silbert, Soft Matter, 2010, 6, 2918.

26. M. Otsuki, H. Hayakawa, and S. Luding, Prog. Theor. Phys. Suppl., 2010, 184, 110-133.

27. M. P. Ciamarra, R. Pastore, M. Nicodemi, and A. Coniglio, Phys. Rev. E, 2011, 84, 1-10.

28. M. Grob, C. Heussinger, and A. Zippelius, Phys. Rev. E, 2014, 89, $2-$ 5.

29. J. Sun and S. Sundaresan, J. Fluid Mech., 2011, 682, 590-616.

30. H. Hwang and K. Hutter, Contin. Mech. Thermodyn., 1995, 7, 357384.

31. P. Johnson, P. Nott, and R. Jackson, J. Fluid Mech, 1990, 210, 501535.

32. D. Berzi, C. di Prisco, and D. Vescovi, Phys. Rev. E, 2011, 84, 1-6.

33. S. Ji and H. H. Shen, J. Rheol., 2008, 52, 87-103.

105 34. S. Chialvo and S. Sundaresan, Phys. Fluids, 2013, 25, 070603.

35. G. Koval, J.-N. Roux, A. Corfdir, and F. Chevoir, Phys. Rev. E, 2009, 79, 021306.

36. S. Chapman and T. G. Cowling, The mathematical theory of nonuniform gases, third edition, Cambridge University Press, 1970.

110 37. C. S. Campbell, J. Fluid Mech., 2002, 465, 261-291.

38. D. Berzi and J. T. Jenkins, Phys. Fluids, 2015, 27, 113303.

39. J. T. Jenkins and C. Zhang, Phys. Fluids, 2002, 14, 1228.

40. M. Larcher and J. T. Jenkins, Phys. Fluids, 2013, 25, 113301.

41. M. W. Richman, Acta Mech., 1988, 75, 227-240.

115 42. J. T. Jenkins and E. Askari, J. Fluid Mech., 1991, 223, 497-508.

43. J. T. Jenkins and O. D. L. Strack, Mech. Mater., 1993, 16, 25-33.

44. D. Howell, R. Behringer, and C. Veje, Phys. Rev. Lett., 1999, 82, 5241-5244.

45. L. D. Landau and E. M. Lifschitz, Theory of elasticity, 1959.

120 46. S. F. Foerster, M. Y. Louge, H. Chang, and K. Allia, Phys. Fluids, 1994, 6, 1108.

47. T. Komatsu, S. Inagaki, N. Nakagawa, and S. Nasuno, Phys. Rev. Lett., 2001, 86, 1757-1760.

48. K. Kamrin and G. Koval, Phys. Rev. Lett., 2012, 108, 178301. 125 49. G. D. R. Midi, Eur. Phys. J. E. Soft Matter, 2004, 14, 341-65. 
50. R. Seto, R. Mari, J. F. Morris, and M. M. Denn, Phys. Rev. Lett., 2013, 111, 1-5.

51. C. Heussinger, Phys. Rev. E, 2013, 88, 050201

52. E. Brown and H. M. Jaeger, J. Rheol., 2012, 56, 875.

5 53. H. Eyring and J. Hirschfelder, J. Phys. Chem., 1937, 41, 249-257. 